\title{
Mathematical Formulation of Relationship between Applied Marketing Effort and Potential Ability of Determining Market Share
}

\author{
Mokhtar M. Metwally \\ School of Economics and Finance, University of Western Sydney, Sydney, NSW 1797, Australia \\ Correspondence should be addressed to Mokhtar M. Metwally, m.metwally@uws.edu.au
}

Received 28 October 2007; Revised 23 December 2007; Accepted 10 March 2008

Recommended by Jack Penm

\begin{abstract}
The aim of this paper is to formulate the mathematical relationship between firms potential ability and their applied efforts to attract the body of unattached customers. A method is devised in this paper by which management techniques imposed by a particular firm can evaluate its market share. This paper demonstrates the relationship between the applied marketing effort of management and the potential ability of the firm in determining its market share. This paper also investigates the effect of a number of simultaneous marketing impulses on the movement of the body of unattached customers and hence on the size of the market share.
\end{abstract}

Copyright (C) 2008 Mokhtar M. Metwally. This is an open access article distributed under the Creative Commons Attribution License, which permits unrestricted use, distribution, and reproduction in any medium, provided the original work is properly cited.

\section{Introduction}

The study of competition among the few has opened two important (related) fields of research: nonprice competition and customer loyalty in local and global markets. These have been the results of work of many researchers specialized in the field of marketing management. This work includes research by Brunner [1], Taylor and Shaw [2], Heflebrower [3], Glusser [4], Drucker [5], Quelch and Hoff [6], Farber and Wycoff [7], Jaworski and Kohli [8], Clancy and Shulman [9], Ambler [10], Mohammed et al. [11], and Berdrow and Lane [12].

When only two or three firms dominate the market (a situation which is very common in many markets for manufactured consumer goods), consumer loyalty is particularly important for a firm that aims at gaining the loyalty of a certain segment of the market. This segment will not (in the short-run and in the absence of cut throat competition) move to another competitor. We have, therefore, loyal customers who are attached to a particular brand [13-15]; and Brown 2001. The implication of this is obvious. If a new firm is to enter a particular market (or an 
existing firm is to expand its market share), the strategy would be to attract the unattached (floating or indifferent customers). Hence, the body of unattached customers would be moving towards some firm depending on the marketing effort used [16-18].

Only some part of the firms potential marketing ability may be in motion. The firm may still be able to inject some new brands or embark on an intensified advertising campaign or improve the quality of its product [19-21]. Hence, there are a number of impulses that can be applied to move the body of unattached customers towards the firms in question.

\section{The mathematical relationship of management evaluation of potential capacity in determining market share}

The position of the body of unattached customers is determined by the magnitudes of a number of independent variables.

Let $(x, y, z)$ be these variables, where

(i) $x$ is say, the firm's level of advertising expenditure;

(ii) $y$ is the number of the firm's brands;

(iii) $z$ is a some quality variable.

These three variables can be expressed in a specified number of independent variables $\theta, \Phi, \Psi, \ldots$, whereby if $t$ is the time, then

$$
\begin{aligned}
& x=f(t, \theta, \Phi, \Psi, \ldots), \\
& y=f(t, \theta, \Phi, \Psi, \ldots), \\
& z=f(t, \theta, \Phi, \Psi, \ldots) .
\end{aligned}
$$

Equations (2.1)-(2.3) may explicitly contain $t$, but they must not contain derivatives of $\theta, \Phi, \Psi, \ldots$ or any other differential coefficients with respect to time $t$.

Now, the body of unattached customers $(m)$ at a certain speed may be expressed mathematically as

$$
F_{t}=m \frac{d v}{d t}
$$

where

$$
d s=v d t,
$$

$v$ being the speed, which can be measured by changes in sales to unattached customers $(s)$ with respect to time:

$$
v=\frac{d s}{d t} .
$$

It follows that

$$
F_{t}=m \frac{d}{d t}\left(\frac{d s}{d t}\right)=m \frac{d^{2} s}{d t^{2}}=m v \frac{d s}{d t} .
$$


An increase in production speed with a reduction in relative sales prices will reduce the percentage of unattached customers.

Integrating from $A_{1}$ where $s=s_{1}$ and $v=v_{1}$ to $A_{2}$, where $s=s_{2}$ and $v=v_{2}$, we write

$$
\begin{gathered}
\int_{\mathrm{S}_{1}}^{S_{2}} F_{t} d s=m, \\
\int_{v_{1}}^{v_{2}} d v=\frac{1}{2} m v_{2}^{2}-\frac{1}{2} m v_{1}^{2} .
\end{gathered}
$$

The left-hand side represents the effect $U_{1 \rightarrow 2}$ of the effort $F$, exerted on the attraction of unattached customers during the changes in market position from $A_{1}$ to $A_{2}$. The expression $1 / 2 m v_{1}^{2}$ is defined here as the applied marketing effort and is denoted by $T$,

$$
T=\frac{1}{2} m v_{2}^{2}
$$

In an effort to maintain its market share, the firm's effective marketing efforts match the competitors' efforts.

Now, let $V$ be the firm's marketing potentiality, where $V$ is a function of $\theta, \Phi, \Psi$, and so on, we have

$$
\begin{aligned}
& \sum m\left(x^{\bullet \bullet} \delta x+\delta y^{\bullet \bullet} \delta y+z^{\bullet \bullet} \delta z\right)=-V \\
& \delta V=\frac{\partial V}{\partial \theta} \delta \theta+\frac{\partial V}{\partial \Phi} \delta \Phi+\frac{\partial V}{\partial \Psi} \delta \Psi+\cdots \\
& \delta x=\frac{\partial x}{\partial \theta} \delta \theta+\frac{\partial x}{\partial \Phi} \delta \Phi+\frac{\partial x}{\partial \Psi} \delta \Psi+\cdots
\end{aligned}
$$

and similar equations for $\delta y$ and $\delta z$.

Noting that

$$
\begin{gathered}
\sum m\left(x^{\bullet \bullet} \frac{\partial x}{\partial \theta}+y^{\bullet \bullet} \frac{\partial y}{\partial \theta}+z^{\bullet \bullet} \frac{\partial z}{\partial \theta}\right)=\frac{\partial V}{\partial \theta} \\
\sum m\left(x^{\bullet \bullet} \frac{\partial x}{\partial \Phi}+y^{\bullet \bullet} \frac{\partial y}{\partial \Phi}+z^{\bullet \bullet} \frac{\partial z}{\partial \Phi}\right)=-\frac{\partial V}{\partial \Phi} \\
\sum m\left(x^{\bullet \bullet} \frac{\partial x}{\partial \Psi}+y^{\bullet \bullet} \frac{\partial y}{\partial \Psi}+z^{\bullet \bullet} \frac{\partial z}{\partial \Psi}\right)=-\frac{\partial V}{\partial \Psi} .
\end{gathered}
$$

If we now differentiate (2.1) we get

$$
x^{\bullet} \frac{\partial x}{\partial t}+\frac{\partial x}{\partial \theta} \theta^{\bullet}+\frac{\partial x}{\partial \Phi} \Phi^{\bullet}+\frac{\partial x}{\partial \Psi} \Psi^{\bullet}+\cdots
$$

Therefore,

$$
\begin{gathered}
\frac{\partial x^{\bullet}}{\partial \theta^{\bullet}}=\frac{\partial x}{\partial \theta} \\
\frac{d}{d t}\left(\frac{\partial x}{\partial \theta}\right)=\frac{\partial^{2} x}{\partial \theta \partial t}+\frac{\partial^{2} x}{\partial \theta^{2}} \theta^{\bullet}+\frac{\partial^{2} x}{\partial \theta \partial \Phi} \Phi^{\bullet}+\frac{\partial^{2} x}{\partial \theta \partial \Psi} \Psi^{\bullet}+\cdots \\
=\frac{\partial}{\partial \theta}\left(\frac{\partial x}{\partial t}+\frac{\partial x}{\partial \theta} \theta^{\bullet}+\frac{\partial x}{\partial \Phi} \Phi^{\bullet}+\frac{\partial x}{\partial \Psi} \Psi^{\bullet}+\cdots\right)=\frac{\partial x^{\bullet}}{\partial \theta} .
\end{gathered}
$$


Noting that

$$
x^{\bullet \bullet} \frac{\partial x}{\partial \theta}=\frac{d}{d t}\left(x^{\bullet} \frac{\partial x}{\partial \theta}\right)-x^{\bullet} \frac{d}{d t} \frac{\partial x}{\partial \theta}=\frac{d}{d t}\left(x^{\bullet} \frac{\partial x^{\bullet}}{\partial \theta^{\bullet}}\right)-x^{\bullet} \frac{d}{d t} \frac{\partial x^{\bullet}}{\partial \theta^{\bullet}}=\frac{d}{d t}\left(x^{\bullet} \frac{\partial x^{\bullet}}{\partial \theta^{\bullet}}\right)-x^{\bullet} \frac{\partial x^{\bullet}}{\partial \theta^{\bullet}}
$$

Then,

$$
\sum m\left[x^{\bullet \bullet} \frac{\partial x}{\partial \theta}+y^{\bullet \bullet} \frac{\partial y}{\partial \theta}+z^{\bullet \bullet} \frac{\partial z}{\partial \theta}\right]=\sum m\left[x^{\bullet \bullet} \frac{\partial x^{\bullet}}{\partial \theta^{\bullet}}+y^{\bullet \bullet} \frac{\partial y}{\partial \theta^{\bullet}}+z^{\bullet \bullet} \frac{\partial z}{\partial \theta^{\bullet}}\right] .
$$

From (2.11) and (2.15),

$$
\begin{gathered}
\frac{d}{d t} \sum m\left[\left(x^{\bullet} \frac{\partial x^{\bullet}}{\partial \theta^{\bullet}}\right)+y^{\bullet} \frac{\partial y^{\bullet}}{\partial \theta^{\bullet}}+z^{\bullet} \frac{\partial z^{\bullet}}{\partial \theta^{\bullet}}\right]-\sum m\left[x^{\bullet} \frac{d}{d t}\left(\frac{\partial x^{\bullet}}{\partial \theta^{\bullet}}\right)+y^{\bullet} \frac{d}{d t}\left(\frac{\partial y^{\bullet}}{\partial \theta^{\bullet}}\right)+z^{\bullet} \frac{d}{d t}\left(\frac{\partial z^{\bullet}}{\partial \theta^{\bullet}}\right)\right] \\
=\frac{d}{d t} \sum m\left[x^{\bullet} \frac{\partial x^{\bullet}}{\partial \theta^{\bullet}}+y^{\bullet} \frac{\partial y^{\bullet}}{\partial \theta^{\bullet}}+z^{\bullet} \frac{\partial z^{\bullet}}{\partial \theta^{\bullet}}\right]-\sum m\left[x^{\bullet} \frac{d}{d t}\left(\frac{\partial x}{\partial \theta}\right)+y^{\bullet} \frac{d}{d t}\left(\frac{\partial y}{\partial \theta}\right)+z^{\bullet} \frac{d}{d t}\left(\frac{\partial z}{\partial \theta}\right)\right] \\
=\frac{d}{d t} \sum m\left[x^{\bullet} \frac{\partial x^{\bullet}}{\partial \theta^{\bullet}}+y^{\bullet} \frac{\partial y^{\bullet}}{\partial \theta^{\bullet}}+z^{\bullet} \frac{\partial z^{\bullet}}{\partial \theta^{\bullet}}\right]-\sum m\left[x^{\bullet} \frac{\partial x^{\bullet}}{\partial \theta^{\bullet}}+y^{\bullet} \frac{\partial y^{\bullet}}{\partial \theta^{\bullet}}+z^{\bullet} \frac{\partial z^{\bullet}}{\partial \theta^{\bullet}}\right]=-\frac{\partial V}{\partial \theta}
\end{gathered}
$$

In virtue of (2.9), we have

$$
\frac{\partial T}{\partial \theta}=\sum m\left[x^{\bullet 2}+y^{\bullet 2}+z^{\bullet 2}\right]
$$

Therefore,

$$
\begin{aligned}
& \frac{\partial T}{\partial \theta}=\sum m\left[x^{\bullet} \frac{\partial x^{\bullet}}{\partial \theta}+y^{\bullet} \frac{\partial y^{\bullet}}{\partial \theta}+z^{\bullet} \frac{\partial z^{\bullet}}{\partial \theta}\right] \\
& \frac{\partial T}{\partial \theta}=\sum m\left[x^{\bullet} \frac{\partial x^{\bullet}}{\partial \theta^{\bullet}}+y^{\bullet} \frac{\partial y^{\bullet}}{\partial \theta^{\bullet}}+z^{\bullet} \frac{\partial z^{\bullet}}{\partial \theta^{\bullet}}\right] .
\end{aligned}
$$

Hence, from (2.19), (2.21), we obtain

$$
\frac{d}{d t}\left(\frac{\partial T}{\partial \theta^{\bullet}}\right)-\frac{\partial T}{\partial \theta}=-\frac{\partial V}{\partial \theta} .
$$

Similarly,

$$
\begin{gathered}
\frac{d}{d t}\left(\frac{\partial T}{\partial \Phi^{\bullet}}\right)-\frac{\partial T}{\partial \Phi}=-\frac{\partial V}{\partial \Phi} \\
\frac{d}{d t}\left(\frac{\partial T}{\partial \Psi^{\bullet}}\right)-\frac{\partial T}{\partial \Psi}=-\frac{\partial V}{\partial \Psi} .
\end{gathered}
$$

Equations (2.22), (2.23) express the relationship between potential marketing ability and applied effort affecting the firm's market share as determined by sales to unattached customers. The same relationship can be investigated when a number of simultaneous 
impulses (e.g., embarking on new advertising campaign, injecting of new brands, and introducing a substantial change in the quality of the product) are applied by the firm to its marketing efforts.

The sum of the virtual moments of the components of changes in sales to unattached customers would be equal to

$$
\sum m\left[x_{1}^{\bullet}-x_{0}^{\bullet}\right] \delta x+\left[y_{1}^{\bullet}-y_{0}^{\bullet}\right] \delta y+\left[z_{1}^{\bullet}-z_{0}^{\bullet}\right] \delta z .
$$

We have

$$
\frac{\partial T}{\partial \theta^{\bullet}}=\sum m\left[x^{\bullet} \frac{\partial x^{\bullet}}{\partial \theta^{\bullet}}+y^{\bullet} \frac{\partial y^{\bullet}}{\partial \theta^{\bullet}}+z^{\bullet} \frac{\partial z^{\bullet}}{\partial \theta^{\bullet}}\right]
$$

Let $T_{0}$ and $T_{1}$ be values of $T$ just before and just after the impulses. Then,

$$
\begin{gathered}
2 T_{0}=\sum m\left[x_{0}^{2 \bullet}+y_{0}^{2 \bullet}+z_{0}^{2 \bullet}\right] \\
\frac{\partial T}{\partial \theta^{\bullet}}=\sum m\left[x^{\bullet}\left(\frac{\partial x^{\bullet}}{\partial \theta^{\bullet}}\right)_{0}+y^{\bullet}\left(\frac{\partial y^{\bullet}}{\partial \theta^{\bullet}}\right)_{0}+z^{\bullet}\left(\frac{\partial z^{\bullet}}{\partial \theta^{\bullet}}\right)_{0}\right] .
\end{gathered}
$$

Now,

$$
\begin{aligned}
\sum m\left[x_{0}^{\bullet} \delta x\right. & \left.+y_{0}^{\bullet} \delta y+z_{0}^{\bullet} \delta z\right] \\
=\sum m & \left\{\left[x_{0}^{\bullet}\left(\frac{\partial x}{\partial \theta}\right)_{0}+y_{0}^{\bullet}\left(\frac{\partial y}{\partial \theta}\right)_{0}+z_{0}^{\bullet}\left(\frac{\partial z}{\partial \theta}\right)_{0}\right]_{0} \delta+\left[x_{0}^{\bullet}\left(\frac{\partial x}{\partial \Phi}\right)_{0}+y_{0}^{\bullet}\left(\frac{\partial y}{\partial \Phi}\right)_{0}+z_{0}^{\bullet}\left(\frac{\partial z}{\partial \Phi}\right)_{0}\right] \delta \Phi\right. \\
& \left.+\left[x_{0}^{\bullet}\left(\frac{\partial x}{\partial \Psi}\right)_{0}+y_{0}^{\bullet}\left(\frac{\partial y}{\partial \Psi}\right)_{0}+z_{0}^{\bullet}\left(\frac{\partial z}{\partial \Psi}\right)_{0}\right] \delta \Psi\right\} .
\end{aligned}
$$

By referring to (2.15), it can be readily shown that

$$
\left(\frac{\partial x^{\bullet}}{\partial \theta^{\bullet}}\right)_{0}=\left(\frac{\partial x}{\partial \theta}\right)_{0}, \text { and so on }
$$

Therefore,

$$
\left(\frac{\partial T}{\partial \theta^{\bullet}}\right)_{0}=\sum m\left[x_{0}^{\bullet}\left(\frac{\partial x}{\partial \theta}\right)_{0}+y_{0}^{\bullet}\left(\frac{\partial y}{\partial \theta}\right)_{0}+z_{0}^{\bullet}\left(\frac{\partial z}{\partial \theta}\right)_{0}\right] .
$$

There will also be similar expression for

$$
\left(\frac{\partial T}{\partial \Phi}\right)_{0}=\left(\frac{\partial T}{\partial \Psi}\right)_{0}, \text { and so on. }
$$

Therefore,

$$
\sum m\left[x_{0}^{\bullet} \delta x+y_{0}^{\bullet} \delta y+z_{0}^{\bullet} \delta z\right]=\left(\frac{\partial T}{\partial \theta^{\bullet}}\right)_{0} \delta \theta+\left(\frac{\partial T}{\partial \Phi^{\bullet}}\right)_{0} \delta \Phi+\left(\frac{\partial T}{\partial \Psi^{\bullet}}\right)_{0} \delta \Psi+\cdots
$$


Similarly, it can be shown that

$$
\sum m\left[x_{0}^{\bullet} \delta x+y_{0}^{\bullet} \delta y+z_{0}^{\bullet} \delta z\right]=\left(\frac{\partial T}{\partial \theta_{1}^{\bullet}}\right) \delta \theta+\left(\frac{\partial T}{\partial \Phi_{1}^{\bullet}}\right)_{0} \delta \Phi+\left(\frac{\partial T}{\partial \Psi_{1}^{\bullet}}\right)_{0} \delta \Psi+\cdots
$$

From (2.24), (2.31), and (2.32),

$$
\begin{aligned}
\sum m & {\left[x_{1}^{\bullet}-x_{0}^{\bullet}\right] \delta x+\left[y_{1}^{\bullet}-y_{0}^{\bullet}\right] \delta y+\left[z_{1}^{\bullet}-z_{0}^{\bullet}\right] \delta z } \\
& =\left(\frac{\partial T_{1}}{\partial \theta_{1}^{\bullet}}-\frac{\partial T_{0}}{\partial \theta_{0}^{\bullet}}\right) \delta \theta+\left(\frac{\partial T_{1}}{\partial \Phi_{1}^{\bullet}}-\frac{\partial T_{0}}{\partial \Phi_{0}^{\bullet}}\right) \delta \Phi+\left(\frac{\partial T_{1}}{\partial \Psi_{1}^{\bullet}}-\frac{\partial T_{0}}{\partial \Psi_{0}^{\bullet}}\right) \delta \Psi+\cdots
\end{aligned}
$$

Let

$$
\sum\left(\frac{\partial T_{1}}{\partial \theta_{1}^{\bullet}}-\frac{\partial T_{0}}{\partial \theta_{0}^{\bullet}}\right) \delta \theta=\sum\left(\frac{\partial T}{\partial \Phi_{1}^{\bullet}}\right)_{0}^{2}
$$

If $X, Y$, and $Z$ are the components of the applied impulse at $(x, y, z)$ then

$$
\sum(X \delta x+Y \delta y+Z \delta z)=P \delta \theta+Q \delta \Phi+R \delta \Psi
$$

Therefore,

$$
\begin{aligned}
& \left(\frac{\partial T}{\partial \theta^{\bullet}}\right)_{1}-\left(\frac{\partial T}{\partial \theta^{\bullet}}\right)_{0}=\left(\frac{\partial T}{\partial \theta^{\bullet}}\right)_{0}^{1}=P, \\
& \left(\frac{\partial T}{\partial \Phi^{\bullet}}\right)_{1}-\left(\frac{\partial T}{\partial \Phi^{\bullet}}\right)_{0}=\left(\frac{\partial T}{\partial \Phi^{\bullet}}\right)_{0}^{1}=Q \\
& \left(\frac{\partial T}{\partial \Psi^{\bullet}}\right)_{1}-\left(\frac{\partial T}{\partial \Psi^{\bullet}}\right)_{0}=\left(\frac{\partial T}{\partial \Psi^{\bullet}}\right)_{0}^{1}=R .
\end{aligned}
$$

$P, Q$, and $R$ are called the generalized components of marketing impulse, while $\left(\partial T / \partial \theta^{\bullet}\right) / \delta \theta^{\bullet}$ is called generalized component of marketing effort.

\section{Conclusions}

This paper has devised a method by which a particular firm can evaluate its potential capacity in determining its market share.

The relationship between the applied marketing effort and the potential ability of the firm determining its market share has also been demonstrated.

The paper also investigated the effect of a number of simultaneous marketing impulses on the movement of body of unattached customers and hence the size of the market shares.

\section{References}

[1] E. Brunner, "Competition and the theory of the firm," Economica Internationale, vol. 5, no. 3, pp. 21-32, 1952.

[2] W. J. Taylor and R. T. Shaw Jr., Marketing: An Internal Analytical Approach, Irwin, Cincinnati, ohio, USA, 1961. 
[3] R. B. Heflebrower, "Competition: static and dynamic," in Planning and Problem Solving in Marketing, W. Alderson and P. E. Green, Eds., Irwin, Homewood, Ill, USA, 1964.

[4] R. Glusser, The New High Priesthood, Macmillan, London, UK, 1967.

[5] P. Drucker, Management Tasks, Responsibilities, Practices, Harper \& Row, New York, NY, USA, 1973.

[6] J. A. Quelch and E. J. Hoff, "Customizing global marketing," Harvard Business Review, pp. 174-183, May-June 1986.

[7] B. Farber and J. Wycoff, "Customer service: evolution and revolution," Sales and Marketing Management, pp. 42-49, May 1991.

[8] P. J. Jaworski and A. K. Kohli, "Market orientation: antecedents and consequences," Journal of Marketing, vol. 57, no. 3, pp. 53-70, 1993.

[9] K. J. Clancy and R. Shulman, "Breaking the mold," Sales and Marketing Management, pp. 82-84, January 1994.

[10] T. Ambler, Marketing and the Bottom Line: The New Metrics of Corporate Wealth, Financial Times PrenticeHall, London, UK, 2000.

[11] R. Mohammed, R. Fisher, B. J. Jaworski, and A. Cahill, Internet Marketing: Building Advantage in a Networked Economy, McGraw-Hill/Irwin, New York, NY, USA, 2002.

[12] I. Berdrow and H. W. Lane, "International joint ventures: creating value through successful knowledge management," Journal of World Business, vol. 38, no. 1, pp. 15-30, 2003.

[13] F. J. Kote, "Market entry and the character of competition," Western Economic Journal, pp. 48-59, December 1966.

[14] J. Thomas, "Quantifying the black economy: 'measurement without theory' yet again?" The Economic Journal, vol. 109, no. 456, pp. 381-389, 1999.

[15] R. Fletcher and L. Brown, International Marketing, Prentice-Hall, Sydney, Australia, 2nd edition, 2002.

[16] M. M. Metwally, "The entry gap in New Zealand markets for manufactured goods," Economic Record, vol. 49 , no. 4, pp. 575-587, 1973 .

[17] M. M. Metwally, "Economic strategies of firms facing asymptotic demand: a case study," Applied Economics, vol. 5, no. 4, pp. 271-280, 1974.

[18] M. M. Metwally, "Determinants of growth of advertising expenditure on consumer goods and services," Journal of International Marketing and Marketing Research, vol. 22, no. 3, pp. 147-156, 1997.

[19] R. Dorfman and P. O. Steinner, "Optimal advertising and optimal quality," in Mathematical Models and Methods in Marketing, F. M. Bass, R. D. Bussell, M. R. Greene, et al., Eds., Irwin, Homewood, Ill, USA, 1961.

[20] S. K. Gupta and K. S. Krishnan, "Mathematical models in marketing," Operations Research, vol. 15, no. 6, pp. 1040-1050, 1967.

[21] M. M. Metwally and H. U. Tamaschke, "Advertising and the propensity to consume," Oxford Bulletin of Economics and Statistics, vol. 43, no. 3, pp. 273-286, 1981. 


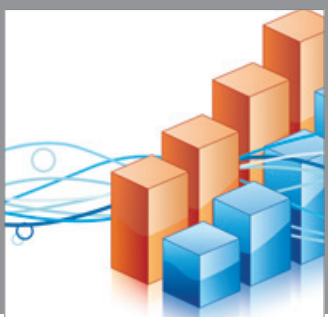

Advances in

Operations Research

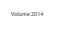

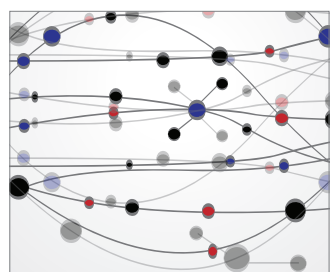

\section{The Scientific} World Journal
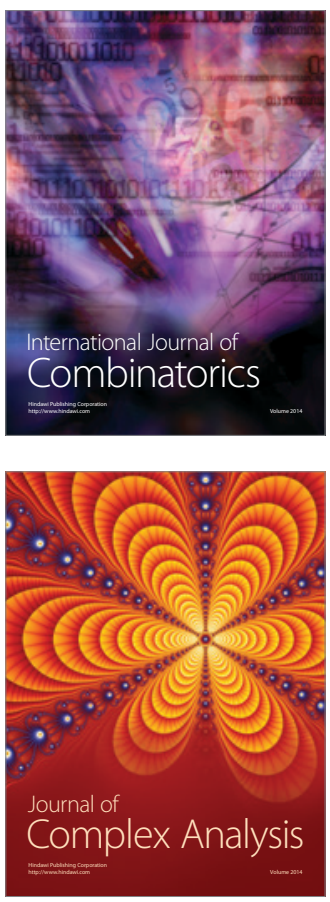

International Journal of

Mathematics and

Mathematical

Sciences
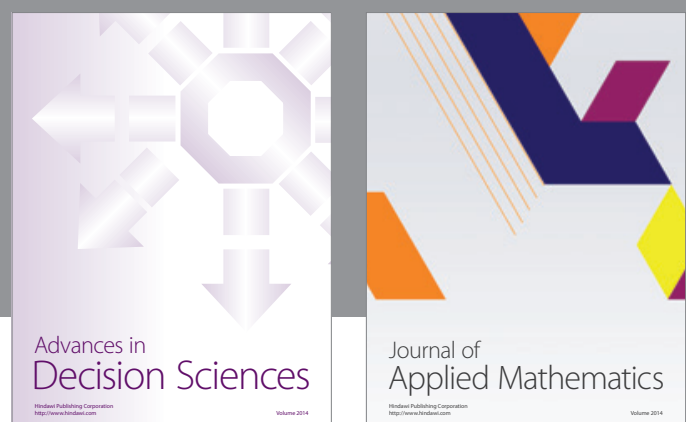

Journal of

Applied Mathematics
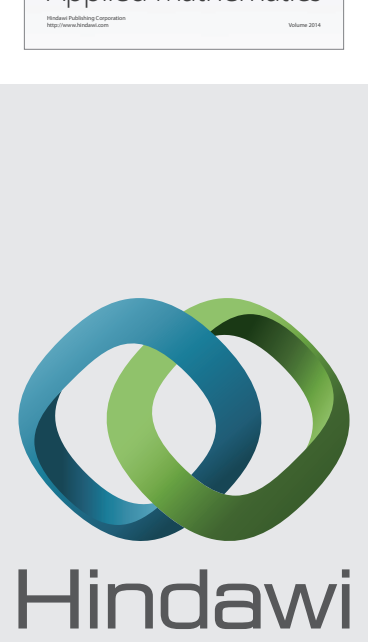

Submit your manuscripts at http://www.hindawi.com
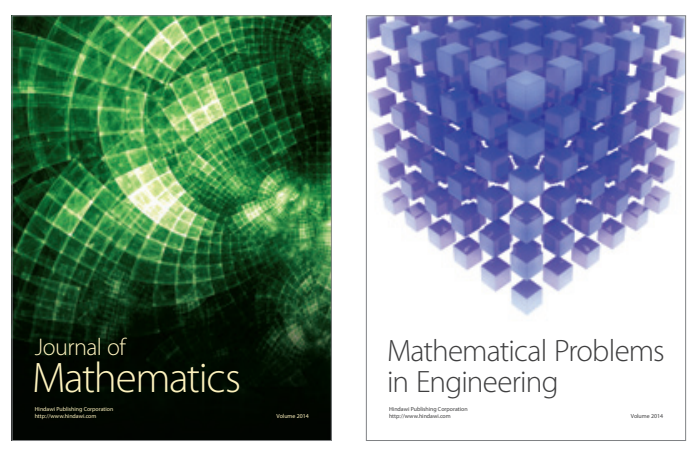

Mathematical Problems in Engineering
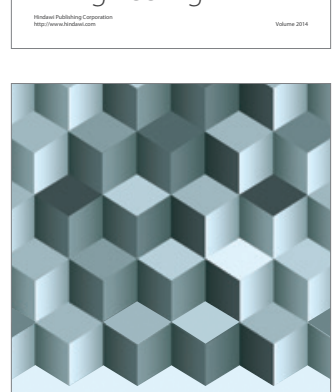

Journal of

Function Spaces
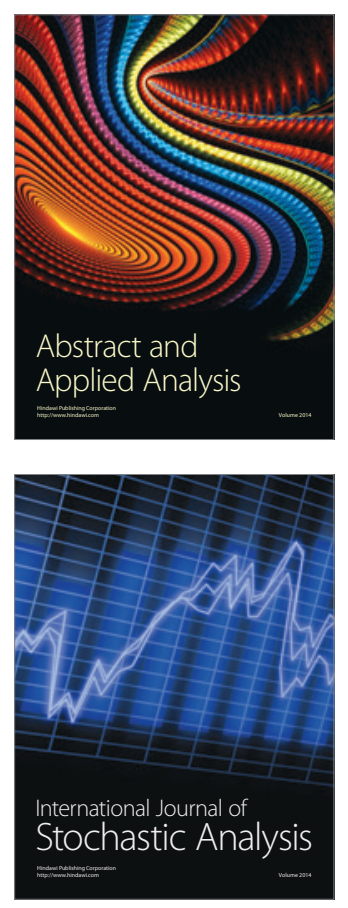

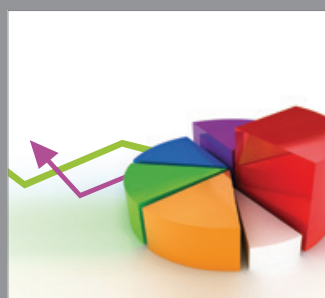

ournal of

Probability and Statistics

Promensencen
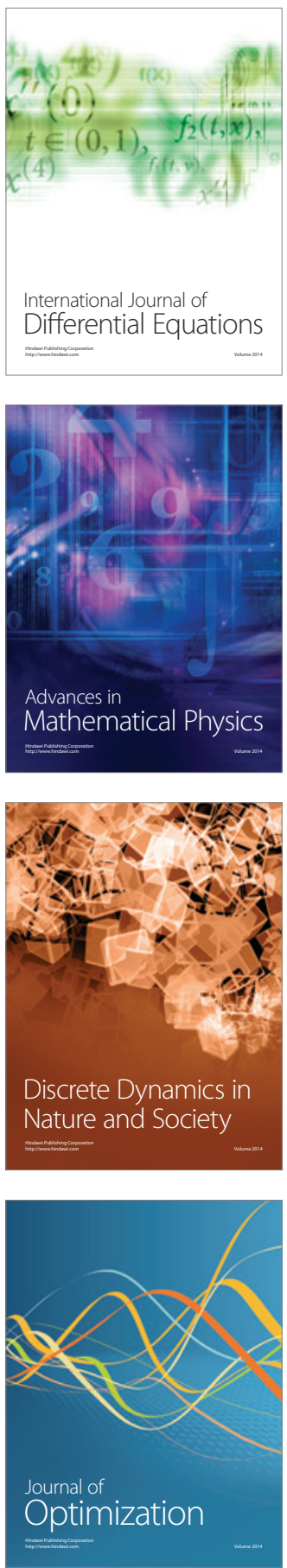